\title{
VOLUME INEQUALITIES FOR ORLICZ MEAN ZONOIDS
}

\section{Changmin Du, Gangsong Leng and Dongmeng XI}

Abstract. In this paper, a more general mean zonoid called Orlicz mean zonoid $\bar{Z}_{\phi} K$ of a convex body $K$ is introduced. Using shadow systems of convex bodies, we give a sharp lower estimate for the volume ratio of $\bar{Z}_{\phi} K$ and $K$, and a sharp upper estimate for the volume product of $\bar{Z}_{\phi}^{*} K$ and $K$.

Mathematics subject classification (2010): 52A20, 52A40.

Keywords and phrases: Convex body, Orlicz mean zonoid, shadow systems.

\section{REFERENCES}

[1] S. CAmpi And P. Gronchi, The L $L^{p}$-Busemann-Petty centroid inequality, Adv. Math. 167 (2002), $128-141$.

[2] S. CAmpi And P. GRonchi, On the reverse $L^{p}$-Busemann-Petty centroid inequality, Mathematika 49 (2002), 1-11.

[3] S. CAmPI And P. Gronchi, On volume product inequalities for convex sets, Proc. Amer. Math. Soc. 134 (2006), 2393-2402.

[4] S. CAmpi And P. GRonchi, Volume inequalities for $L^{p}$-zonotopes, Mathematika 53 (2006), 71-80.

[5] R. J. Gardner AND G. Zhang, Affine inequalities and radial mean bodies, Amer. J. Math. 120 (1998), 505-528.

[6] R. J. Gardner, Geometric Tomography, 2nd edition, Encyclopedia of Mathematics and its Applications, vol. 58, Cambridge University Press, Cambridge, 2006.

[7] R. J. Gardner, D. Hug AND W. WeIL, The Orlicz-Brunn-Minkowski theory: A general framework, additions, and inequalities, J. Differential Geom., accepted.

[8] P. M. Gruber, Convex and Discrete Geometry, Grundlehren Math. Wiss., vol. 336, Springer, Berlin, 2007.

[9] C. HABerL, $L_{p}$ intersection bodies, Adv. Math. 217 (2008), 2599-2624.

[10] C. Haberl And F. Schuster, General $L_{p}$ affine isoperimetric inequalities, J. Differential Geom. 83 (2009), 1-26.

[11] C. Haberl and F. Schuster, Asymmetric affine $L_{p}$ Sobolev inequalities, J. Funct. Anal. 257 (2009), 641-658.

[12] C. Haberl, E. Lutwak, D. Yang And G. Zhang, The even Orlicz Minkowski problem, Adv. Math. 224 (2010), 2485-2510.

[13] Q. Huang And B. He, On the Orlicz Minkowski problem for polytopes, Discrete Comput. Geom. 48 (2012), 281-297.

[14] A. Li And G. Leng, A new proof of the Orlicz Busemann-Petty centroid inequality, Proc. Amer. Math. Soc. 139 (2011), 1473-1481.

[15] M. Ludwig, Minkowski valuations, Trans. Amer. Math. Soc. 357 (2005), 4191-4213.

[16] M. Ludwig And M. Reitzner, A classification of $S L(n)$ invariant valuations, Ann. Math. 172 (2010), 1219-1267.

[17] E. LutwaK, The Brunn-Minkowski-Firey theory. I. Mixed volumes and the Minkowski problem, J. Differential Geom. 38 (1993), 131-150.

[18] E. LutwaK, The Brunn-Minkowski-Firey theory. II. Affine and geominimal surface areas, Adv. Math. 118 (1996), 244-294. 
[19] E. LutwaK, D. YANG AND G. ZhANG, $L_{p}$ affine isoperimetric inequalities, J. Differential Geom. 56 (2000), 111-132.

[20] E. Lutwak, D. Yang And G. Zhang, $L_{p}$ John ellipsoids, Proc. London Math. Soc. 90 (2005), 497-520.

[21] E. LutwaK, D. Yang And G. Zhang, Orlicz projection bodies, Adv. Math. 223 (2010), 220-242.

[22] E. Lutwak, D. YAng And G. Zhang, Orlicz centroid bodies, J. Differential Geom. 84 (2010), 365-387.

[23] C. M. PetTy, Ellipsoids, Convexity and its Applications (P. M. Gruber and J. M. Wills, eds.), Birkhäuser, Basel, 1983, pp. 264-276.

[24] R. E. PFIEFER, The Extrema of Geometric Mean Values, PhD dissertation, Department of Mathematics, University of California, Davis, CA, 1982.

[25] R. E. PFIEFER, Maximum and minimum sets for some geometric mean values, J. Theoret. Probab. 3 (1990), 169-179.

[26] C. A. Rogers And G. C. Shephard, Some extremal problems for convex bodies, Mathematika 5 (1958), 93-102.

[27] R. SCHNEIDER, Random hyperplanes meeting a convex body, Z. Wahr. Verw. Gebiete 61 (1982), 379-387.

[28] R. Schneider And W. Weil, Zonoids and related topics, Convexity and its Applications, Birkhäuser, Basel, 1983, pp. 296-317.

[29] R. SchneIder, Convex Bodies: The Brunn-Minkowski Theory, Cambridge Univ. Press, Cambridge, 1993.

[30] G. C. ShePhard, Shadow systems of convex bodies, Israel J. Math. 2 (1964), 229-236.

[31] J. SteIneR, Einfacher Beweis der isoperimetrischen Hauptsätze, J. Reine Angew Math. 18 (1838), 281-296, and Gesammelte Werke Vol. 2, 77-91, Reimer, Berlin, 1882.

[32] G. WAng, G. Leng And Q. HuAng, Volume inequalities for Orlicz zonotopes, J. Math. Anal. Appl. 391 (2012), 183-189.

[33] M. Weberndorfer, Shadow systems of asymmetric L L zonotopes, Adv. Math. 240 (2013), 613635.

[34] G. Zhang, Restricted chord projection and affine inequalities, Geom. Dedicata 39 (1991), 213-222. 